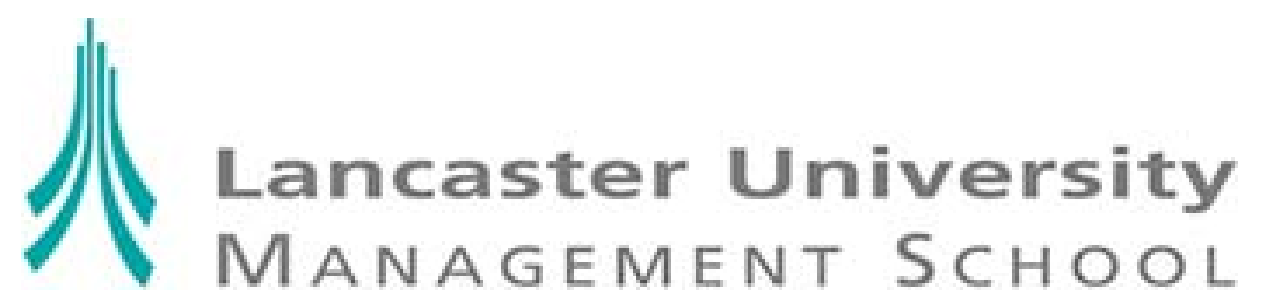

\author{
Lancaster University Management School \\ Working Paper \\ 2003/089
}

\title{
On the use and improvement of Hull and White's control variate technique
}

\section{Mark Shackleton and San-Lin Chung}

The Department of Accounting and Finance

Lancaster University Management School

Lancaster LA1 4YX

UK

CMark Shackleton and San-Lin Chung

All rights reserved. Short sections of text, not to exceed two paragraphs, may be quoted without explicit permission, provided that full acknowledgement is given. 


\title{
On the use and improvement of Hull and White's control variate technique
}

\author{
San-Lin Chung ${ }^{1} \quad$ Mark Shackleton ${ }^{2}$
}

July 2003

${ }^{1}$ Department of Finance, National Taiwan University, Taipei, Taiwan, R.O.C.

${ }^{2}$ Department of Accounting and Finance, Lancaster University, U.K. Tel: 44-1524594131, Email: m.shackleton@lancaster.ac.uk. 


\begin{abstract}
Our article provides a study on the use and improvement of Hull and White's (1988) control variate technique in pricing options. It contributes to the literature in two ways. Firstly we show that it is not optimal to use the entire error of a control variate against its known price (usually a closed-form solution) to correct and improve the unknown error of the unknown price of a complex option and we derive a better error correction fraction. Secondly while Hull and White only advocated the use of the simplest European option control variate, we show how to choose better controls to reduce pricing errors more effectively and we discuss the role of so called static hedges as the best theoretical control variates.
\end{abstract}

\title{
JEL Classification: G13
}

Keywords: control variate, American option, exponential exercise boundary, static hedge. 


\section{Introduction}

Many problems in finance must ultimately be solved through the use of a numerical technique as the only practical and universally robust solution method. By numerical technique, we mean that the state space in question must be partitioned and discretized in some manner (either using a tree or a grid) before the value of the asset is evaluated at each and every of the many resultant points. Moreover these points (tree nodes or grid points) have to be chosen before the prices at those points are known.

In the field of options and particularly for American options ${ }^{1}$ (where no closed form solution is generally available), such a numerical brute force method is necessary because it is generally the only way to guarantee that increasing computational effort results in increased numerical accuracy. Other areas where numerical techniques are necessary include all dynamic programming techniques where the value of a strategy must be evaluated simultaneously with its optimal action, for instance in optimal portfolio control under transaction costs and similar problems.

It is important to be able to determine the price of a financial claim to arbitrary accuracy, firstly because a financial intermediary may be concerned about overpaying or undercharging. Secondly and more importantly, because the financial intermediary is often left with the task of replicating the claim over its life, he must be able to evaluate the comparative statics (the hedge parameters or so called Greeks of the option) repeatedly and quickly if he is to respond to revised hedging needs. For exotic options (especially path dependent options), this task may be sensitive to the method chosen and remaining errors will be a function of the computational time spent (see Boyle and Lau (1994) and Figlewski and Gao (1999)).

\footnotetext{
${ }^{1}$ Asian options and other exotics also fall into this category.
} 
Although several approximations ${ }^{2}$ are available for American options (also for Asians), many of which have the right asymptotic properties and converge to the true price in the limit of one variable, these do not allow the price of a general option to be determined to arbitrary accuracy. Even if a series solution is actually available for pricing an option to arbitrary accuracy (e.g. Geske and Johnson (1984)), it may still be more efficient ${ }^{3}$ to employ a numerical method compared to the task of evaluating a complex series. This is because the required multivariate cumulative normal function (and other high dimensional numerical integrations on which many compound options solutions rely) although expressed as an integral and computer coded very efficiently, is not strictly a closed form solution in that it requires a numerical method itself for computation. Arbitrary accuracy is only achieved through increasing computational power and so other methods are typically preferred to the multivariate cumulative normal function.

Furthermore, the numerical methods themselves are subject to various problems. The rate of convergence of the estimated price to the true price, may not be fast and the convergence may not be monotone in that the sign and magnitude of the error may oscillate as a finer time grid or tree is used (see Figure 1). This is because there are two sources of discretization error.

Firstly, discretization at the terminal boundary will yield imperfect prices. Interpolation techniques and trapezoidal integration rules can minimize these errors for smooth payoff functions but the first derivative at the final exercise point of an option may not be smooth so the grid or lattice density here is critical. ${ }^{4}$

\footnotetext{
${ }^{2}$ Approximate solutions for American options include the work of Geske and Johnson (1984), MacMillan (1986), Barone-Adesi and Whalley (1987), Omberg (1987), and Ju (1998) etc. Although quick to evaluate, none of these methods will allow accuracy to be increased with more computational effort.

${ }^{3}$ Here, efficient means that the method takes less computational time for the same accuracy or gives more accurate estimates for the same computational time.

${ }^{4}$ Figlewski and Gao (1999) propose a method termed Adaptive Mesh Model (AMM) to
} 
Secondly, the chosen numerical array must be used to evaluate the point of optimal action simultaneously with the desired prices. This will involve further forms of discretization error, one in determining the location of the optimal exercise points itself and one when subsequently valuing the option earlier in time, which is dependent on the value at, and position of, the optimal exercise boundary (Wilmott, Howison and Dewynne (1995) detail a complimentary condition that can reduce this boundary error). This will cause problems particularly if the American exercise boundary is far from linear or if it is discontinuous at maturity.

This is to say that the critical points in the option pricing problem (that coincide with an optimal exercise boundary) are as important as the option prices away from the boundary. Indeed the boundary conditions have equal standing with the asset partial differential equation itself in pricing the claim because it is the boundary conditions alone that distinguish one type of option claim from another. It is the (prior) positioning of the nodes in state space relative to these critical thresholds in the option pricing problem that cause the second type of pricing error. Furthermore it is the non-linear way that the state space grid or tree interacts with a boundary of general shape that causes these errors to depend chaotically ${ }^{5}$ on the grid size (see Figure 1). A small error in the placement of the grid next to a boundary can lead to a large pricing error. By way of example, Boyle and Lau (1994) show that convergence is very slow when using binomial methods to price Barrier options because of the severe nonlinearity present as the mesh or lattice points oscillate around the boundary reduce the "nonlinearity error" at the terminal boundary. In their method, one or more small sections of fine high-resolution lattice are added onto a tree with coarser time and price steps. This will incorporate the more accurate fine-mesh values for the critical nodes into the coarse tree and thus reduce the nonlinearity error.

${ }^{5} \mathrm{~A}$ chaotic variable is one that could be evaluated exactly with sufficient computing power; without such resource it may appear to be "random" (i.e. there may be no apparent short cut to its determination). Since the emphasis here is to use as little computational power as possible, we will treat these chaotic errors as if they were truely random (i.e. without deterministic form). 
with decreasing mesh size.

One way to reduce these types of errors and hence improve the computational time required is the so called "control variate" method proposed by Hull and White (1988, hereafter HW). They suggested that a similar claim for which a closed form solution is available be priced on the same grid and made subject to the same discretization errors. Comparison of the resultant price with the analytic price is instructive for two reasons, firstly the magnitude of the errors against the known price may be related to the errors on the unknown price, secondly the sign of the two may be related. In their paper, the use of a Black Scholes control spared the use of a finer grid and thus more computation.

In addition to HW, this paper contributes to the literature in two directions. Firstly HW used $100 \%$ of the error in this control variate against its known price to correct and improve the unknown error of the unknown price. We will show that the full use of the control variable may not be optimal. In some cases using the entire error to correct the unknown error will increase rather than decrease the latter.

Secondly it is interesting to note that HW only advocated the use of a simple control variate, the corresponding Black and Scholes European option price. This will help deal with the first type of discretization error at maturity but will not necessarily help with the second source of error in the location of and value near the boundary. We suggest other instruments that may well help in the second area.

The rest of this paper is organized as follows. Section 2 discusses the methodology and the sources of pricing errors when using discrete lattice methods. Section 3 presents the numerical results and suggests how to choose good controls to effectively correct errors. Section 4 concludes this article. 


\section{Methodology}

Numerical methods based on a finite number of calculations will always contain error, in particular for binomial methods only for an arbitrarily large number of tree nodes will the binomial price converge to the true continuous price. That is for a given set of option parameters (e.g. stock, strike, risk free, volatility, maturity) $\theta=\left(S_{0}, X, r, \sigma, T\right)$ and number of time steps $n$ implemented in the tree, the estimated option price $\hat{P}(\theta, n)$ will only converge to the true price $P(\theta)=\lim \hat{P}(\theta, n)$ as $n \rightarrow \infty$. As mentioned previously, even then there is a problem since convergence to the limit may be oscillatory.

We can define an option error $\varepsilon(n)$ for any given numerical method or choice of $n$, the magnitude of which should decrease as $n \rightarrow \infty$

$$
\varepsilon(n)=\hat{P}(\theta, n)-P(\theta) \quad \lim _{n \rightarrow \infty} \varepsilon(n)=0
$$

The control variate $(\mathrm{CV})$ technique is applicable when there are two similar ${ }^{6}$ options, labelled $C$ and $T$. The Target option $T$ is the (difficult) option which we want to price while $C$ is a Control option, called a Control Variate (or CV), which is similar to option $T$ but has an analytical (or closed-form) solution $P_{C}$. To apply the control variate technique, the values of $T$ and $C$ have to be evaluated using the same numerical procedure (i.e. with the same value of $n$ if trees are used). The numerical price estimates of $T$ and $C$ are denoted as $\hat{P}_{T}$ and $\hat{P}_{C}$, respectively and they depend on $n$. The control variate estimate for the value of $T, \widetilde{P}_{T}$, is given by

$$
\begin{aligned}
\widetilde{P}_{T} & =\hat{P}_{T}+c\left(P_{C}-\hat{P}_{C}\right) \\
\varepsilon_{C} & =\hat{P}_{C}-P_{C}
\end{aligned}
$$

\footnotetext{
${ }^{6}$ By similar options we mean that both option prices should follow the same partial differential equation (e.g. European vs. American options), both option payoffs should be highly correlated (geometric average vs. arithmetic average Asian options) or that their boundary conditions should be congruent (continuously vs. discretely monitored barrier options).
} 


$$
\begin{aligned}
& \varepsilon_{T} \approx c \varepsilon_{C} \\
& \widetilde{P}_{T}=\hat{P}_{T}-c \varepsilon_{C} .
\end{aligned}
$$

This is to say that the error of the control option $\varepsilon_{C}$ can be used to improve the error of the target option $\varepsilon_{T}$ because it may be closely linked to it.

Hull and White recommended the use of a corresponding Black Scholes European option price as a control variable $P_{C}$ for an American option and they then used the pricing error of the discretized price estimate on the European to improve the American, i.e. the entire error $c=1$ of the control variate to correct the unknown error of the price estimate of the target option.

Without any prior knowledge of the dependency between the target errors and the control errors, the $c=1$ assumption is probably best but examination of actual error dependency may yield a different error sensitivity and potentially a better use of the control. The idea is to always chose the value of that leads to the minimum target error, at least over a limited range of estimations $n$.

This then leads to the question of how to estimate the dependency between the control and target errors. This could be examined across different methods, but within a particular numerical method such as binomial trees we investigate the errors as a function of different tree step numbers $n$ indexed by $i$. We always do this for the same parameter set $\theta$ and generate option price estimates $\hat{P}(\theta, i)$ for many $i$. For a given set of target option inputs, it is not desirable to include control errors from other parameter input combinations $\theta^{\prime}$ (since they may not respond/correlate in the same manner) so instead we employ other numerical estimate errors across different $i$ but for the same $\theta$. The idea is to use a relatively small number of time steps $i$ between two reference levels $l, m$ to determine the target/control error dependency quickly and then use this to apply to a much larger tree of size $n$ that is only evaluated once $(l<m \ll n)$.

Option prices for the control and target will be evaluated for different numbers of time steps between $l, m$ inclusive (i.e. a total of $m-l+1$ ) in order to determine 
the correlation coefficient and optimal control ratio. This optimal control ratio will then be applied to errors from the control and target calculated from a much larger tree with $n \gg m, l$ in order to obtain a more accurate estimate of the limiting value $n \rightarrow \infty$.

If we wish to minimize the error variance of $\widetilde{P}_{T}$ then the optimal correction to use is

$$
\begin{aligned}
c^{*}(l, m) & =\operatorname{Cov}(T, C) / \operatorname{Var}(C)=\rho(T, C) \sigma_{T} / \sigma_{C} \\
& =\frac{\sum_{i=l}^{m} \varepsilon_{C}(i) \varepsilon_{T}(i)}{\sum_{i=l}^{m} \varepsilon_{C}^{2}(i)}
\end{aligned}
$$

where $\rho(T, C)$ is the correlation coefficient between $\hat{P}_{T}$ and $\hat{P}_{C}, \sigma_{T}^{2}\left(\sigma_{C}^{2}\right)$ is the variance of $\hat{P}_{T}\left(\hat{P}_{C}\right)$, and $c^{*}$ is the optimal coefficient in which case the variance of the estimate will be minimized at

$$
\operatorname{Var}\left(\hat{P}_{T}+c^{*}\left(P_{C}-\hat{P}_{C}\right)\right)=\left(1-\rho(l, m)^{2}\right) \sigma_{T}^{2},
$$

a quantity that could be zero if the correlation were perfect.

Most importantly, it will not generally be the case that the HW choice of $c=1$ will yield the best results, especially not if $\rho(T, C)<<1$. In fact if this is the case, the resulting error may well be greater if $c=1$ is used, and clearly a variate with $\rho(T, C)=0$ cannot help improve pricing. This is the case in Figure 1 where the American pricing error is plotted as a function of the number of grid points used. As can be seen the errors using $c=1$ can actually be greater than using $c=0$ (i.e. no control is used). ${ }^{7}$ Figure 2 shows a scatter graph of the American option pricing errors as a function of the European errors, i.e. a visualisation of the correlation and slope coefficients used in this paper, the dependency between the two is clear from this figure.

\section{Figures 1 and 2 ABOUT HERE}

\footnotetext{
${ }^{7}$ Note that $c^{*}$ can in fact be greater than one if the errors in $T$ exceed the errors in $C$ and the correlation is high, i.e. the variance of the target option price must be greater than that of the control price, a situation which may be possible for barrier options controlled with standard European options.
} 
There are in fact two attributes of the control variate error that matter for improving target prices, one is the magnitude of the its pricing errors and the second is the correlation of its errors with those on the unknown price of the target option.

This paper is concerned with the choice of control variate, the correlation of target errors and finally the magnitude of the errors that result from an optimal use of the $\mathrm{CV}$ technique. We examine a range of control variates, the correlation of their errors and their ability to enhance pricing.

We propose evaluating the performance of CV techniques under $c=1$ and $c^{*}(l, m)$ methods in order to evaluate the performance of the Black Scholes (BS) control variate. We also propose evaluating the performance of other control variates that may reduce discretization error near an exercise boundary. For example, the analytical approximations of Barone-Adesi, Whaley (1987), MacMillan (1986) (BAWM) and Omberg (1984) (Om) both allow determination of an approximate (but not optimal) exercise boundary that can be used to correct the discretization error near the true exercise boundary. For each and every array location, it is possible to say if it lies in or outside the boundary as approximated by each of the formulae. Thus the evaluation of these methods under grid pricing and closed form may yield extra information about the nature of the discretization error near a boundary that is a reasonable approximation for the true boundary. The performance of these new forms compared to existing control variates will elucidate something of the nature of the discretization errors that are incurred at different points of the grid.

For each CV (European, Omberg options etc.) the optimal $c^{*}(l, m)$ is established as follows. We calculate the prices of the control option and the target option using one lattice method, e.g. simple binomial method, for a reasonable number of time steps, say $l=20$ to $m=50$ steps. We then obtain two series of prices which allow us to estimate their variance-covariance and hence $c^{*}(l, m)$. We then use a much larger tree $(n=10,000)$ and the prices it generates for 
both target and control along with the optimal $c^{*}(l, m)$ use of these controls.

\section{$3 \quad$ Numerical Results}

The most efficient use of a control variate is not necessarily $c=1$, for a given step size estimation and use of $c^{*}$ will generally improve price estimates. However it takes time to estimate $c^{*}$, therefore it is important whether $c^{*}$ can be estimated using only a few samples (Monte Carlo simulation) or time steps (binomial method). We estimate $c^{*}$ as follows. We calculate the prices of the target option and the control option using binomial trees using different numbers of time steps, for example, $l=20$ to $m=100$, this generates two series of prices which allow us to estimate $c^{*}(l, m)$. In Table 1 , the target options are American put options and the control options are their counterpart European options. Table 1 shows that the estimates of $c^{*}$ are quite similar for different ranges of time steps. In other words, we can estimate $c^{*}$ using only few (e.g. 20 to 40) time steps but apply it for trees with more time steps. Therefore it is beneficial to use $c^{*}$ rather than the prior of $c=1$ because it takes little computational time to estimate it.

\section{Table 1 ABOUT HERE}

To compute the benchmark values of the American options used in validating the approximation methods, we first investigate the accuracy of four lattice approaches; the binomial method of Cox, Ross, and Rubinstein (1979), the binomial Black and Scholes (BBS) method and the binomial Black and Scholes method with Richardson extrapolation (BBSR) of Broadie and Detemple (1996), ${ }^{8}$ and the Adaptive Mesh Model (AMM) of Figlewski and Gao (1999) (see footnote 4). We follow the procedure in Broadie and Detemple $(1996,1997)$

\footnotetext{
${ }^{8}$ The BBS method is a modification of the binomial method where the Black-Scholes formula replaces the usual "continuation value" at the last time step just before option maturity.
} 
to run a comparison. We first choose a large set of options $\left(3^{5}=243\right.$ options; 3 values of each of 5 variables) with parameters of practical interest (parameter values from other work). Then for each method we price the test set of options and compute percentage error measures. The root-mean-squared (RMS) relative percentage error is used as the error measure and is defined by:

$$
\mathrm{RMS}=\sqrt{\frac{1}{T} \sum_{j=1}^{T} e_{j}^{2}}
$$

where $T=243$ is the number of options under consideration, $e_{j}=\left(\widehat{P}_{j}\left(\theta_{j}\right)-\right.$ $\left.P_{j}\left(\theta_{j}\right)\right) / P_{j}\left(\theta_{j}\right)$ is the relative error ${ }^{9}$ of the $j$-th option, $P_{j}\left(\theta_{j}\right)$ is the true option price (Black-Scholes), $\widehat{P}_{j}\left(\theta_{j}\right)$ is the estimated option price.

The results (Table 2) indicate that the BBSR method produces the most accurate values of these four methods. Therefore, we use the BBSR with $n=10,000$ time steps to produce benchmark values for American options in the following numerical evaluations.

\section{Table 2 ABOUT HERE}

We define $c^{*}(l, m)$ as the optimal coefficient of the target and control option estimated from using the binomial prices with few time steps, $l$ to $m(l<m)$. Treating this numerical experiment as a statistical experiment, when evaluating pricing errors for a number of tree steps between $l, m$ we borrowing terminology and label $c^{*}(l, m)$ as an in sample coefficient. This coefficient is used as a proxy

The BBSR method adds Richardson extrapolation to the BBS method. In particular, the BBSR method with $n$ steps computes the corresponding BBS price for $n / 2$ steps $P(\theta, n / 2)$ and $n$ steps $P(\theta, n)$ and then sets the BBSR approximation to the asymptotic price equal to $P(\theta, \infty)=2 P(\theta, n)-P(\theta, n / 2)$.

${ }^{9}$ Note that these percentage errors are calculated using the same number of steps $(n=10,000)$ but across different parameter sets $j$ i.e. $e(j)=$ $(\widehat{P}(\theta(j), i)-P(\theta(j), i)) / P(\theta(j), i)$ as oppose to the previous absolute errors which were calculated for the same parameter set $\theta$ but for different number of tree steps $i$ i.e. $\epsilon(i)=$ $\widehat{P}(\theta, i)-P(\theta, i)$ for $i=l$ to $m$. 
for the unknown out of sample coefficient $c^{*}(l, n)$ and the errors used in making this assumption are evaluated.

Table 3 shows that the CV technique can significantly reduce the RMS relative errors of price estimates for the binomial method. The CV technique using $c^{*}$ improves the RMS relative error by about 20 to 25 times for the binomial method. Combining with the results (their Figures 2 to 8) of Broadie and Detemple (1996), we can find that the binomial method coupled with the CV technique using $c^{*}$ seems more efficient than other proven (numerically) efficient methods such as the BBSR method. Moreover, Table 3 suggests that CV technique using $c^{*}$ works well for both in-sample and out-of-sample tests. ${ }^{10}$

\section{Table 3 ABOUT HERE}

Another objective of the CV technique is actually to seek a better instrument (with closed or analytic form pricing solution) whose numerical errors have as high a correlation as possible with the target option prices errors and then use these as corrections. To date the CV technique has used Black-Scholes (since its errors are positively correlated because a deep out of the money American, is priced as a European anyway) but other closed form formulae or indeed approximating formulae could be used instead. For instance Omberg (1987) has a closed form expression for American option prices assuming an exponential boundary (since this boundary is not actually optimal the formula will undervalue options), this boundary can be numerically applied and a binomial price for Omberg compared to the theoretical Omberg price. It may not matter that the Omberg price is not actually an optimal price of an option ${ }^{11}$, what matters is that its errors may correlate very well with the true American price errors ${ }^{12}$.

\footnotetext{
${ }^{10}$ Because $c^{*}$ is estimated using binomial trees with 20 to 100 time steps, we call 30, 50, and 80 time steps in-sample test, and 200, 400, and 600 time steps out-of-sample test.

${ }^{11}$ although it corresponds to the price of a claim with an arbitrary, but optimised, boundary shape.

${ }^{12}$ the option bias between the actual and binomial Omberg prices should be small.
} 
This is so because its arbitrary boundary does not get too far from the true one and it will suffer errors in the same regions as the true American (near the boundary).

To verify the above argument, we compare the performance of European options with that of Omberg options. Table 4 shows that the average correlation coefficients between American options and Omberg options are high (0.974). However, it is a little surprising that the performance of Omberg options ${ }^{13}$ as the controls is only slightly better than European options. A possible explanation is that the oscillating binomial prices may be not able to distinguish a good control from a bad control. ${ }^{14}$ Overall, the results still suggest that an option with wisely ${ }^{15}$ chosen exercise boundary is a good control for American options but that the final maturity boundary errors due to grid point positioning still matter.

\section{Table 4 ABOUT HERE}

Note that the control can be an option or a portfolio of options and theoretically the best control option(s) are those that have the highest correlation coefficient with the price of the target option. Recently the static hedge literature ${ }^{16}$ has been used to replicate an exotic option using a portfolio of standard (closed form)

\footnotetext{
${ }^{13}$ In terms of percentage the absolute values of correlation coefficients of Omberg options are still generally $(65.1 \%)$ higher than those of European options.

${ }^{14}$ One evaluation criterion to determine a good numerical method may be its ability to distinguish good and bad controls. From this point of view, the binomial method may fail in some cases.

${ }^{15}$ meaning that the arbitrary boundary is very close to the true exercise boundary, for example, Ju (1998) proposes a multipiece exponential exercise boundary which can approximate the true boundary much better than Omberg (1987)

${ }^{16}$ The basic principle behind static hedge method is as follows. If the prices of the target option and the static hedge portfolio follow the same partial differential equation and they are worth the same on a certain boundary, they are also worth the same at all interior points of the boundary (see Derman, Ergener, and Kani (1995) or Carr, Ellis, and Gupta (1998) for details).
} 
options. Since an effective static hedge portfolio can approximately replicate the option position, it will also provide an effective control to reduce the variance of the numerical estimates of option prices. $^{17}$

As an example, we investigate the performance of three controls which partially match the boundary conditions of a barrier option (cite for parameters?). The barrier option considered in this paper is an up-and-out call option with $S=$ $100, K=100, H=120$ (barrier), $T=1$ year, $r=0.1$ (annually compounded risk free rate), $q=0.05$ (annually compounded dividend yield), and $\sigma=0.25$ (volatility). The first control (1) is the counterpart European option which exactly matches the boundary if the barrier is never crossed before maturity. The second control (2) matches the boundary of the barrier option at maturity. The third control (3) replicates the value of the barrier option; (a) at expiration below the barrier, and (b) exactly on the 120 barrier at one year and 6 months prior to expiration (half way). It is obvious that portfolio (3) should be a better control than portfolio (1) because it can match more nodes on the boundary of the barrier option. Table 5 indicates that the third control is the best control and can reduce the RMS relative errors by a factor of about three.

Following Derman, Ergener and Kani (1995), one can easily find other control which can match as many nodes on the boundary as desired. ${ }^{18}$ In other words, theoretically one can use the static hedge portfolio to find a perfect control which can correct all the errors although it will involve an increasing number of terms.

\section{Table 5 ABOUT HERE}

\footnotetext{
${ }^{17}$ However it may be very difficult and complicated to formulate a perfect static hedge portfolio in practice thus reducing the benefits of the CV method.

${ }^{18}$ Derman, Ergener, and Kani (1995) have shown that a replicating portfolio using 24 options to match the zero boundary value on the barrier at half-month intervals can hedge the (continuous) barrier option very well.
} 


\section{Conclusion}

This paper has considered the use and improvement of Hull and White's (1988) control variate technique in option pricing. Firstly we discussed the sources of pricing errors when using discrete lattice method and recommended good controls that effectively correct target errors. Secondly, we gave examples that showed that Hull and White's control variate technique is not optimal and potentially it may increase rather than decrease the pricing errors.

To effectively reduce pricing errors, the control variate technique has to be customized to both the type of numerical methods and the options in question. The benefits of this effort are reduced errors and a deeper understanding of their origin and control.

It would be interesting to study the possibility of combining recent advanced numerical methods with the control variate technique to price complex options efficiently. For example, Barraquand and Martineau (1995) and Longstaff and Schwartz (2001) have proposed a method for pricing multidimensional American options by Monte Carlo simulation. Given the flexibility for pricing exotic options under complex asset price processes, combining their methods with the control variate technique may provide a powerful tool for both academics and practitioners. 


\section{References}

Barone-Adesi, G., and R. E. Whaley, 1987, Efficient Analytic Approximation of American Option Values, Journal of Finance, 42, 301-320.

Barraquand J. and D. Martineau, 1995, Numerical valuation of high dimensional multivariate American securities, Journal of Financial and Quantitative Analysis, 30(3), 381-405.

Boyle, P., and S. H. Lau, 1994, Bumping Up Against the Barrier with the Binomial Model, Journal of Derivatives, 1, 6-14.

Broadie, M., and J. Detemple, 1996, American option valuation: new bounds, approximations, and a comparison of existing methods, Review of Financial Studies, 9, 1211-1250.

Carr, P., K. Ellis, and V. Gupta, Static Hedging of Exotic Options, Journal of Finance, 53, 1165-1190.

Cox, J., S. Ross and M. Rubinstein (1979), Option Pricing: A Simplified Approach, Journal of Financial Economics, 7, 229-263.

Derman, E., D. Ergener, and I. Kani, 1995, Static Option Replication, Journal of Derivatives, 2, 78-95.

Figlewski, S., and B. Gao, 1999, The adaptive mesh model: a new approach to efficient option pricing, Journal of Financial Economics, 53, 313-351.

Geske, R., and H. E. Johnson, 1984, The American Put Option Valued Analytically, Journal of Finance, 39, 1511-1524.

Hull, J., and A. White, 1988, The Use of the Control Variate Technique in Option Pricing, Journal of Financial and Quantitative Analysis, 23, 237-251. 
Ju, N., 1998, Pricing an American Option by Approximating Its Early Exercise Boundary as a Multipiece Exponential Function, Review of Financial Studies, 11(3), 627-646.

Longstaff, F. A., and E. S. Schwartz, 2001, Valuing American Options by Simulations: A Simple Least-Squares Approach, The Review of Financial Studies, $14,113-147$.

MacMillan, L. W., 1986, Analytic Approximation for the American Put Option, Advances in Futures and Options Research, 1, 119-139.

Omberg, E., 1987, The Valuation of American Puts with Exponential Exercise Policies, Advances in Futures and Options Research, 2, 117-142.

Wilmott, P., S. Howison, and J. Dewynne, 1995, The Mathematics of Financial Derivatives: A Student Introduction, Cambridge University Press, Cambridge. 
Table 1: Estimated optimal control $c^{*}(l, m)$ values using different sets of time steps

\begin{tabular}{|c|c|c|c|c|c|c|}
\hline & & & \multicolumn{4}{|c|}{$c^{*}(l, m)($ and $\rho(l, m))$} \\
\cline { 4 - 7 }$K$ & $T$ (months) & $\sigma$ & $(20,40)$ & $(20,100)$ & $(150,200)$ & $(250,300)$ \\
\hline 35 & 1 & 0.2 & $0.96(0.999)$ & $0.99(0.999)$ & $0.96(0.998)$ & $0.95(0.999)$ \\
\hline 35 & 1 & 0.3 & $0.95(0.999)$ & $0.98(0.999)$ & $0.96(0.999)$ & $0.95(0.999)$ \\
\hline 35 & 1 & 0.4 & $0.95(0.999)$ & $0.98(0.999)$ & $0.96(0.999)$ & $0.95(0.999)$ \\
\hline 35 & 4 & 0.2 & $0.90(0.990)$ & $0.92(0.989)$ & $0.90(0.993)$ & $0.87(0.994)$ \\
\hline 35 & 4 & 0.3 & $0.88(0.989)$ & $0.92(0.990)$ & $0.88(0.995)$ & $0.90(0.996)$ \\
\hline 35 & 4 & 0.4 & $0.88(0.993)$ & $0.92(0.992)$ & $0.92(0.997)$ & $0.90(0.997)$ \\
\hline 35 & 7 & 0.2 & $0.83(0.984)$ & $0.86(0.983)$ & $0.82(0.991)$ & $0.81(0.994)$ \\
\hline 35 & 7 & 0.3 & $0.82(0.985)$ & $0.87(0.985)$ & $0.87(0.993)$ & $0.84(0.994)$ \\
\hline 35 & 7 & 0.4 & $0.87(0.990)$ & $0.90(0.990)$ & $0.86(0.995)$ & $0.88(0.997)$ \\
\hline 40 & 1 & 0.2 & $0.81(0.999)$ & $0.84(0.995)$ & $0.84(1.000)$ & $0.84(1.000)$ \\
\hline 40 & 1 & 0.3 & $0.86(0.999)$ & $0.89(0.997)$ & $0.88(1.000)$ & $0.88(1.000)$ \\
\hline 40 & 1 & 0.4 & $0.88(1.000)$ & $0.91(0.998)$ & $0.91(1.000)$ & $0.91(1.000)$ \\
\hline 40 & 4 & 0.2 & $0.70(0.998)$ & $0.73(0.991)$ & $0.73(1.000)$ & $0.73(1.000)$ \\
\hline 40 & 4 & 0.3 & $0.77(0.998)$ & $0.80(0.993)$ & $0.80(1.000)$ & $0.80(1.000)$ \\
\hline 40 & 4 & 0.4 & $0.81(0.999)$ & $0.84(0.995)$ & $0.84(1.000)$ & $0.84(1.000)$ \\
\hline 40 & 7 & 0.2 & $0.64(0.998)$ & $0.66(0.991)$ & $0.66(1.000)$ & $0.66(1.000)$ \\
\hline 40 & 7 & 0.3 & $0.72(0.999)$ & $0.75(0.992)$ & $0.75(1.000)$ & $0.75(1.000)$ \\
\hline 40 & 7 & 0.4 & $0.77(0.998)$ & $0.79(0.994)$ & $0.79(1.000)$ & $0.79(1.000)$ \\
\hline 45 & 1 & 0.2 & $0.00(0.000)$ & $0.00(0.000)$ & $0.00(0.000)$ & $0.00(0.000)$ \\
\hline 45 & 1 & 0.3 & $0.51(0.967)$ & $0.51(0.946)$ & $0.57(0.994)$ & $0.57(0.996)$ \\
\hline 45 & 1 & 0.4 & $0.79(0.984)$ & $0.81(0.970)$ & $0.79(0.996)$ & $0.77(0.997)$ \\
\hline 45 & 4 & 0.2 & $0.36(0.931)$ & $0.37(0.933)$ & $0.35(0.977)$ & $0.33(0.974)$ \\
\hline 45 & 4 & 0.3 & $0.63(0.972)$ & $0.68(0.953)$ & $0.67(0.987)$ & $0.67(0.993)$ \\
\hline 45 & 4 & 0.4 & $0.77(0.973)$ & $0.82(0.965)$ & $0.78(0.993)$ & $0.75(0.991)$ \\
\hline 45 & 7 & 0.2 & $0.40(0.899)$ & $0.41(0.918)$ & $0.41(0.976)$ & $0.38(0.983)$ \\
\hline 45 & 7 & 0.3 & $0.64(0.955)$ & $0.68(0.948)$ & $0.65(0.987)$ & $0.64(0.981)$ \\
\hline 45 & 7 & 0.4 & $0.75(0.987)$ & $0.79(0.976)$ & $0.74(0.995)$ & $0.68(0.989)$ \\
\hline
\end{tabular}

The options are puts with other parameters: $S=40, r=0.0488, q=0.0$ (dividend yield). The bracket (e.g. 20,40) means that $c^{*}$ and $\rho$ are estimated using binomial trees with step numbers from 20 to 40 . The correlation coefficients $(\rho)$ are given in parentheses. 
Table 2: The root-mean-squared (RMS) relative errors using the following four methods to price European options

\begin{tabular}{|c|c|c|c|}
\hline binomial & BBS & BBSR & AMM \\
\hline $4.59 \mathrm{E}-05=0.459 \mathrm{bp}$ & $5.41 \mathrm{E}-05=0.541 \mathrm{bp}$ & $2.46 \mathrm{E}-06=0.0246 \mathrm{bp}$ & $2.98 \mathrm{E}-04=2.98 \mathrm{bp}$ \\
\hline
\end{tabular}

The options are European puts. The root-mean-squared relative errors are defined as follows:

$\mathrm{RMS}=\sqrt{\frac{1}{m} \sum_{j=1}^{m} e_{j}^{2}}$

where $e_{j}=\left(P^{*}{ }_{j}-P_{j}\right) / P_{j}$ is the relative error, $P_{j}$ is the accurate option price (Black-Scholes), $P_{j}^{*}$ is the estimated option price. The number of time steps in each method is $n=10,000$. There are 243 sets of parameters are used: $S=40, K=35,40,45, \sigma=0.2,0.3,0.4, T=1,4$, 7 months, $r=3,5,7 \%$, and $q=2,5,8 \%$.

Table 3: The root-mean-squared (RMS) relative errors of price estimates

\begin{tabular}{|l|c|c|c|c|c|c|}
\cline { 2 - 7 } \multicolumn{1}{c|}{} & \multicolumn{3}{c|}{ in-sample test } & \multicolumn{3}{c|}{ out-of-sample test } \\
\cline { 2 - 7 } \multicolumn{1}{c|}{} & 30 & 50 & 80 & 200 & 400 & 600 \\
\hline no CV & $1.74 \mathrm{E}-02$ & $1.46 \mathrm{E}-02$ & $6.38 \mathrm{E}-03$ & $2.38 \mathrm{E}-03$ & $1.88 \mathrm{E}-03$ & $8.11 \mathrm{E}-04$ \\
& $174 \mathrm{bp}$ & $146 \mathrm{bp}$ & $63.8 \mathrm{bp}$ & $23.8 \mathrm{bp}$ & $18.8 \mathrm{bp}$ & $8.11 \mathrm{bp}$ \\
\hline$c=1$ & $1.14 \mathrm{E}-03$ & $6.86 \mathrm{E}-04$ & $5.26 \mathrm{E}-04$ & $1.83 \mathrm{E}-04$ & $9.98 \mathrm{E}-05$ & $6.45 \mathrm{E}-05$ \\
& $11.4 \mathrm{bp}$ & $6.86 \mathrm{bp}$ & $5.26 \mathrm{bp}$ & $1.83 \mathrm{bp}$ & $0.998 \mathrm{bp}$ & $0.645 \mathrm{bp}$ \\
& $(93.4 \%)$ & $(95.3 \%)$ & $(91.8 \%)$ & $(92.3 \%)$ & $(94.7 \%)$ & $(92.0 \%)$ \\
\hline$c^{*}$ & $7.43 \mathrm{E}-04$ & $4.36 \mathrm{E}-04$ & $3.80 \mathrm{E}-04$ & $1.19 \mathrm{E}-04$ & $7.82 \mathrm{E}-05$ & $4.77 \mathrm{E}-05$ \\
& $7.43 \mathrm{bp}$ & $4.36 \mathrm{bp}$ & $3.80 \mathrm{bp}$ & $1.19 \mathrm{bp}$ & $0.782 \mathrm{bp}$ & $0.477 \mathrm{bp}$ \\
& $(95.7 \%)$ & $(97.0 \%)$ & $(94.0 \%)$ & $(95.0 \%)$ & $(95.8 \%)$ & $(94.1 \%)$ \\
\hline
\end{tabular}

The options are American puts. The root-mean-squared relative errors are defined as follows:

$\mathrm{RMS}=\sqrt{\frac{1}{m} \sum_{j=1}^{m} e_{j}^{2}}$

where $e_{j}=\left(P_{j}^{*}-P_{j}\right) / P_{j}$ is the relative error, $P_{j}$ is the accurate American option price (estimated by the BBSR method with 10000 steps), $P_{j}^{*}$ is the estimated option price. There are 243 sets of parameters are used: $S=40, K=35,40,45, \sigma=0.2,0.3,0.4, T=1,4$, 7 months, $r=3,5,7 \%$, and $q=2,5,8 \%$. The coefficient $c *$ is estimated using trees with 20 to 100 steps. The numbers in parentheses are the error reduction percentages using the control variate technique with $c^{*}$ and $c=1$. 
Table 4: The absolute value of correlation coefficients between the American option prices and the control option prices

\begin{tabular}{|c|c|c|}
\hline & European option & Omberg option \\
\hline mean & 0.97246 & 0.97405 \\
\hline max & 0.99967 & 0.99973 \\
\hline min & 0.291753 & 0.32823 \\
\hline
\end{tabular}

The options are American puts. There are 180 sets of parameters are used: $S=40, K=30$ to 50 in increments of $5, \sigma=0.2,0.4,0.6, T=6,12,18,24$ months, $r=3,5,7 \%$, and $q=0$. The correlation coefficient is estimated using binomial trees with 60 to 100 steps. The first control is the counterpart European option with maturity date $T$. The second control is the counterpart Omberg option which has an exponential exercise boundary.

Table 5: The root-mean-squared (RMS) relative errors for the barrier option

\begin{tabular}{c|c|c|c|c} 
& No control & Portfolio 1 & Portfolio 2 & Portfolio 3 \\
\hline RMS & $6.66 \mathrm{E}-03$ & $5.79 \mathrm{E}-03$ & $4.34 \mathrm{E}-03$ & $2.42 \mathrm{E}-03$ \\
& $66.6 \mathrm{bp}$ & $57.9 \mathrm{bp}$ & $43.4 \mathrm{bp}$ & $24.2 \mathrm{bp}$
\end{tabular}

The barrier option is an up-and-out call with $S=100, K=100, H=120, T=1$ year, $r=0.1$ (annually compounded), $q=0.05$ (annually compounded), and $\sigma=0.25$. Portfolio 1 is the counterpart European call option. Portfolio 2 consists of long one European call option with $K=100$, short one European call option with $K=H$, and short one cash-or-nothing (digital) call option with $K=H$ and cash $Q=H-100$. Portfolio 3 is a static hedge portfolio from Derman, Ergener and Kani's (1995) (their Portfolio 4). The root-mean-squared relative errors are defined as follows:

$\mathrm{RMS}=\sqrt{\frac{1}{m} \sum_{j=1}^{m} e_{j}^{2}}$

where $e_{j}=\left(P_{j}^{*}-P_{j}\right) / P_{j}$ is the relative error, $P_{j}$ is the accurate barrier option price (closed-form solution), $P_{j}^{*}$ is the estimated option price using $n_{i}$ steps per half-year, where $n_{i}$ equals to 250 to 300 steps. 
Figure 1: The American put option price with regard to the number of time steps (binomial method)

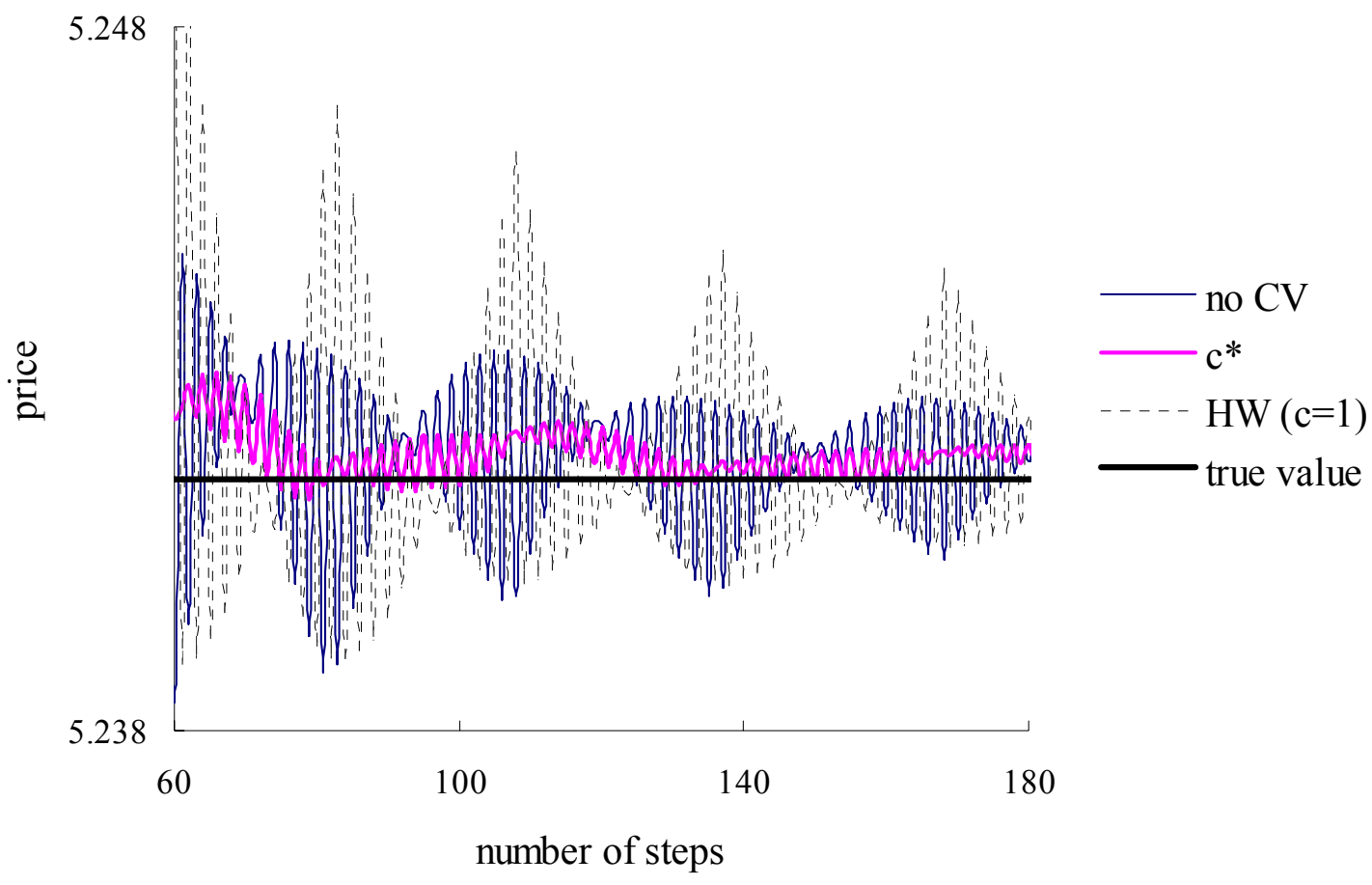

The parameters are $S=40, K=45, T=7$ months, $r=0.07, q=0.02$, and $\sigma=0.2$. The coefficient $c^{*}$ is estimated using binomial trees with 20 to 50 steps. The accurate American option price is estimated using the BBSR method with 10,000 steps. 
Figure 2:

American against European option errors for tree steps between 20 and 100 .

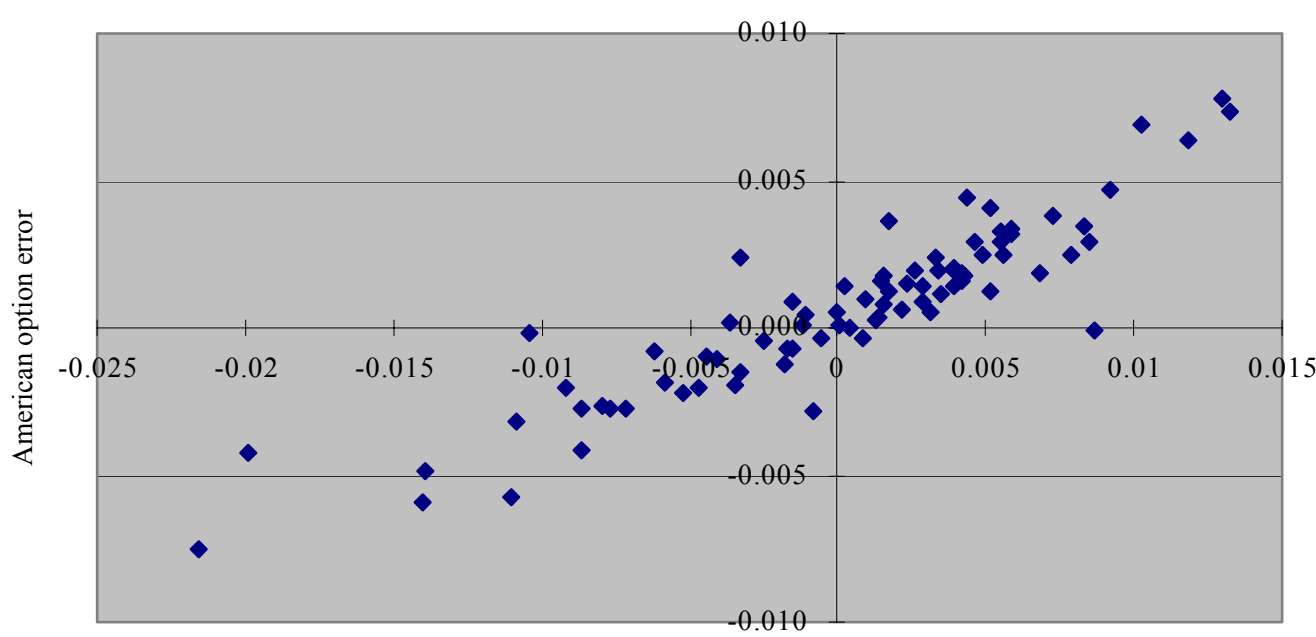

European option error 